\title{
High Data Rate Modulation of High Power 1060-nm DBR Tapered Lasers With Separate Contacts
}

\author{
Pawel Adamiec, Antonio Consoli, Jose Manuel G. Tijero, Santiago Aguilera, Ignacio Esquivias, \\ Sven Schwertfeger, Andreas Klehr, Hans Wenzel, Bernd Sumpf, and Götz Erbert
}

\begin{abstract}
Direct optical modulation at $2.5 \mathrm{~Gb} / \mathrm{s}$ with amplitude of more than $0.5 \mathrm{~W}$ has been demonstrated in single longitudinal mode distributed Bragg reflector tapered lasers emitting at $1060 \mathrm{~nm}$ with separated injection of the ridge waveguide and tapered sections. The modulating signal of $\sim 110 \mathrm{~mA}$ peak to peak was applied to the ridge waveguide section, yielding a high modulation efficiency of $\sim 5 \mathrm{~W} / \mathrm{A}$. The large-signal frequency response of the experimental set-up was limited by the bandwidth of the electrical amplifier rather than by the internal dynamics of the laser, indicating that higher bit rates could be achieved with improved driving electronics.
\end{abstract}

Index Terms-High brightness semiconductor lasers, direct modulation, multi-contact lasers.

\section{INTRODUCTION}

$\mathbf{H}$ IGH speed direct modulation of a high power laser beam is of great interest for several applications, including free-space optical communications [1], laser projection displays [2] and random modulation Continuous Wave (CW) lidar [3]. Tapered lasers with separated electrical access to the ridge waveguide (RW) section and to the tapered section are a promising approach to achieve simultaneously a highbrightness beam and a high speed modulation [4], [5]. This approach has the advantage of controlling the high optical power emitted from the large aperture tapered section with a relatively small electrical current injected into the RW section. Michel et al. [5] reported a very high modulation efficiency (ratio between the increment of the output power and the modulating current swing, $\Delta \mathrm{P} / \Delta \mathrm{I})$ of $20 \mathrm{~W} / \mathrm{A}$ at $700 \mathrm{Mb} / \mathrm{s}$ and 50 W/A for quasi-static modulation, together with a high Optical Modulation Amplitude (OMA) of $1.7 \mathrm{~W}$ and an extinction ratio (ER) of $19 \mathrm{~dB}$. Kwok et al. [6] reported the direct modulation of the RW section in separated contact Distributed Bragg Reflector (DBR) tapered lasers emitting at $1060 \mathrm{~nm}$ up to $1 \mathrm{~Gb} / \mathrm{s}$ and OMA of $0.4 \mathrm{~W}$. The predicted power budget for a Bit Error Rate (BER) of $10^{-9}$ was $47 \mathrm{~dB}$, indicating that the devices were suitable for free-space optical communication systems. In similar devices, a modulation efficiency of 58 W/A was reported in quasi-static modulation conditions at $60{ }^{\circ} \mathrm{C}$ [7].

In this letter we demonstrate the modulation at $2.5 \mathrm{~Gb} / \mathrm{s}$ of two-section tapered lasers with an OMA of $0.53 \mathrm{~W}$. We analyse the origin of the high frequency limit in these devices and conclude that higher data rates together with higher output powers could be achieved by improving the current injection electronics.

\section{DEvice DESCRIPTION AND SETUP}

The laser devices used in this letter have been previously described in [8], and therefore we provide here only a summary of the most important characteristics. The devices are composed of a $1 \mathrm{~mm}$ DBR section which provides an effective reflectivity of around $55 \%$, a $1 \mathrm{~mm} \mathrm{RW}$ section and a $2 \mathrm{~mm}$ tapered section with $214 \mu \mathrm{m}$ output aperture and $0.7 \%$ front facet reflectivity. The vertical laser structure consists of an AlGaAs optical waveguide and three strain-compensated InGaAs quantum wells [9]. The devices were mounted p-side up on standard $\mathrm{C}$-mounts and attached to a copper holder for characterization. The device lay-out was designed for $\mathrm{CW}$ operation and therefore no attention was paid in the design to minimize the electrical parasitics. The mounting allowed the separate driving of the RW and the tapered section currents. A microstrip circuit with a coupling series resistance of $50 \Omega$ was used to supply the high-frequency signal to the RW section.

The output power under $\mathrm{CW}$ operation of both sections was measured using a thermal detector (Gentec UP19K$\mathrm{H})$ placed close to the output facet. The laser was tested under high-speed data modulation with the experimental setup schematically shown in Fig. 1. A Pulse Pattern Generator (PPG) (Anritsu MU181020A) provides a peak-to-peak output voltage $\mathrm{V}_{\mathrm{pp}}$ up to $2.5 \mathrm{~V}$. The generator output was amplified with a 20 dB Radio Frequency (RF) amplifier (Mini-Circuits ZFL-2500 VH+) with maximum electrical power of $+23 \mathrm{dBm}$ and low and high cut-off frequencies of $10 \mathrm{MHz}$ and $2.5 \mathrm{GHz}$, respectively. The amplified data signal was superimposed to a $\mathrm{CW}$ bias current $\mathrm{I}_{\mathrm{RW}}$ through a bias-tee and both were applied 


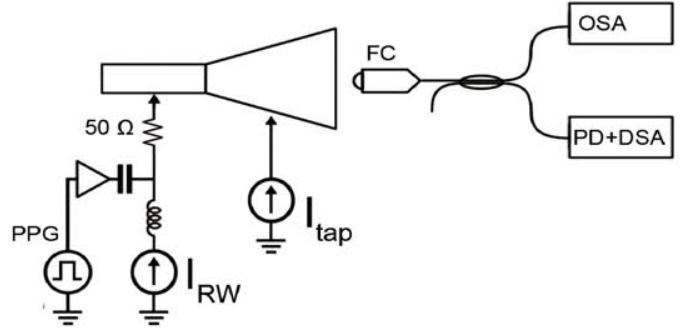

Fig. 1. Schematics of the data modulation experimental set-up. PPG: Pulse Pattern Generator; FC: Fibre collimator; OSA: Optical Spectrum Analyzer; PD: Photodiode; DSA: Digital Signal Analyzer.

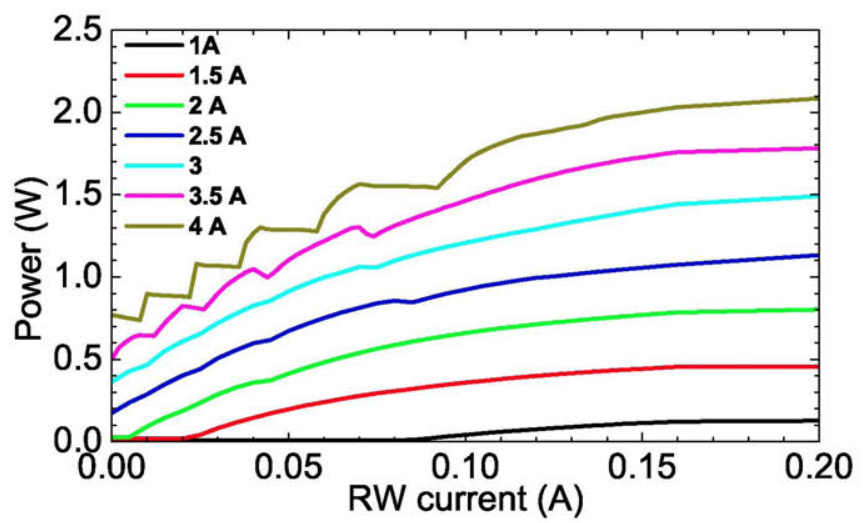

Fig. 2. Output power vs. RW current for different tapered section currents (from bottom to top $\mathrm{I}_{\text {tap }}=1 \mathrm{~A}, 1.5 \mathrm{~A}, 2 \mathrm{~A}, 2.5 \mathrm{~A}, 3 \mathrm{~A}, 3.5 \mathrm{~A}$, and $4 \mathrm{~A}$ ).

to the RW section of the laser. The tapered section was driven in $\mathrm{CW}$ conditions at a current $\mathrm{I}_{\mathrm{tap}}$.

A fraction of the modulated optical power was collected by a fibre collimator. The data signal was measured with the optical module of a $20 \mathrm{GHz}$ Digital Signal Analyzer (DSA) (Tektronix DSA8000) which provided statistical analysis. The absolute power of the modulated optical signal was calibrated by comparing the total power measured with the thermal detector and the fractions of power measured in the DSA when both laser sections were driven in $\mathrm{CW}$ conditions. We ensured that the measured temporal profiles were repetitive and independent of the position of the fiber collimator with respect to the laser facet. All the measurements were performed at a constant temperature of $25{ }^{\circ} \mathrm{C}$.

\section{RESULTS AND DISCUSSION}

Fig. 2 shows the measured optical power $\mathrm{P}$ as a function of the RW section current $I_{R W}$ for different values of the tapered section current $\mathrm{I}_{\text {tap. }}$. The maximum measured output power was $\sim 2 \mathrm{~W}$ at $\mathrm{I}_{\mathrm{RW}}=0.2 \mathrm{~A}_{\text {and }} \mathrm{I}_{\text {tap }}=4 \mathrm{~A}$. At taper section currents higher than $2.1 \mathrm{~A}$ the device shows lasing action even for $\mathrm{I}_{\mathrm{RW}}=0 \mathrm{~A}$. The kinks observed in the P-I $\mathrm{I}_{\mathrm{RW}}$ characteristics (more noticeable at high tapered section currents) are due to mode hopping between the Fabry-Perot cavity modes, as a consequence of cross- and self-thermal detuning of the RW, DBR, and tapered sections [10]. Fig. 2 indicates that the highest quasi-static OMA for this device at room temperature is $1.4 \mathrm{~W}$ for a peak to peak RW current excursion of $0.2 \mathrm{~A}$

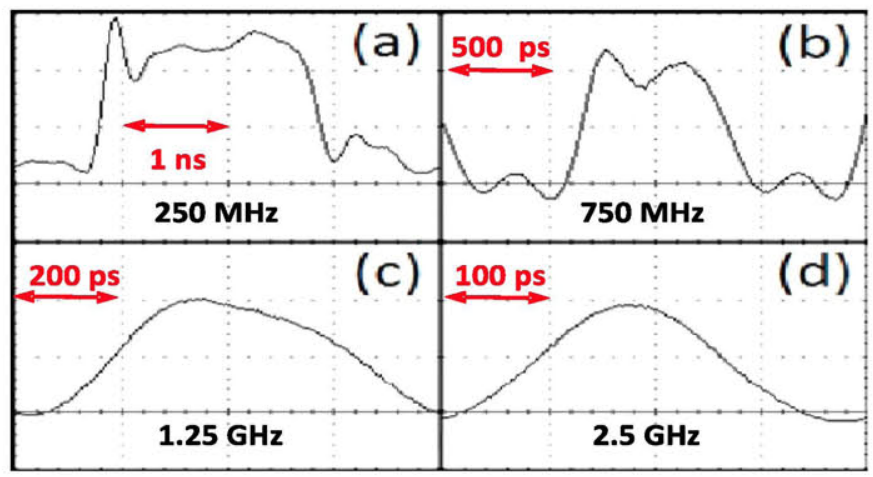

Fig. 3. Traces of the optical response under square modulation at different clock frequencies. The peak to peak PPG output voltage in all the cases was $0.6 \mathrm{~V}$.

at a tapered current of $4 \mathrm{~A}$, with a modulation efficiency of $7 \mathrm{~W} / \mathrm{A}$ and an ER of $4.5 \mathrm{~dB}$.

The laser response was tested under data modulation with a Non-Return to Zero (NRZ) pseudo-random bit sequence $\left(2^{31}-1\right)$ applied to the $\mathrm{RW}$ section at bit rates $\mathrm{B}_{\mathrm{R}}$ ranging from $100 \mathrm{Mb} / \mathrm{s}$ to $7.5 \mathrm{~Gb} / \mathrm{s}$, and also by applying a square signal equivalent to "010101..." bit sequence (equivalent to clock signal at frequency $B_{R} / 2$ ). The dynamic response of the device was characterized by measuring the eye diagram, the quality parameter Q as provided by the DSA, and the OMA when modulating $\mathrm{I}_{\mathrm{RW}}$ with the square signal. The measured signals were not digitally filtered in the DSA.

Optimum CW driving conditions of the RW and tapered sections for high speed modulation and large OMA were found to be $I_{\text {tap }}=4 \mathrm{~A}$ and $\mathrm{I}_{\mathrm{RW}}=120 \mathrm{~mA}$. For these static conditions, Fig. 3 shows the measured optical response under square modulation at different clock frequencies for a peak to peak PPG output voltage of $0.6 \mathrm{~V}$, which corresponds approximately to a peak to peak current excursion of $110 \mathrm{~mA}$. The response at low frequency $(<1 \mathrm{GHz})$ shows clear ringing during both off-on and on-off switching, which are attributed to the combined effects of the poor impedance matching and of the laser relaxation oscillations. At frequencies between $1 \mathrm{GHz}$ and $2.5 \mathrm{GHz}$ an almost sinusoidal waveform is observed. The amplitude of this sinusoidal waveform decreases at higher frequencies due to the combined responses of the laser, the driving circuit, and the electrical amplifier.

The large signal modulation response of the complete system under square modulation was characterized as a function of the clock frequency for different modulation depths in the RW section, with and without the electrical amplifier. Figure 4 shows the normalized OMA as a function of the clock frequency for different modulation depths. The OMA has been normalized with respect to the average of the measured values at frequencies lower than $1 \mathrm{GHz}$. The scattering of the data at low frequencies is not due to noise, but to the ringing of the switching response (see Fig. 3), which leads to fluctuations in the amplitude of the output signal when changing the frequency.

Specifically, Fig. 4 compares the modulation response under two driving conditions of nominally the same modulation depth, namely, $2.5 \mathrm{~V}$ generated directly by the PPG, and 


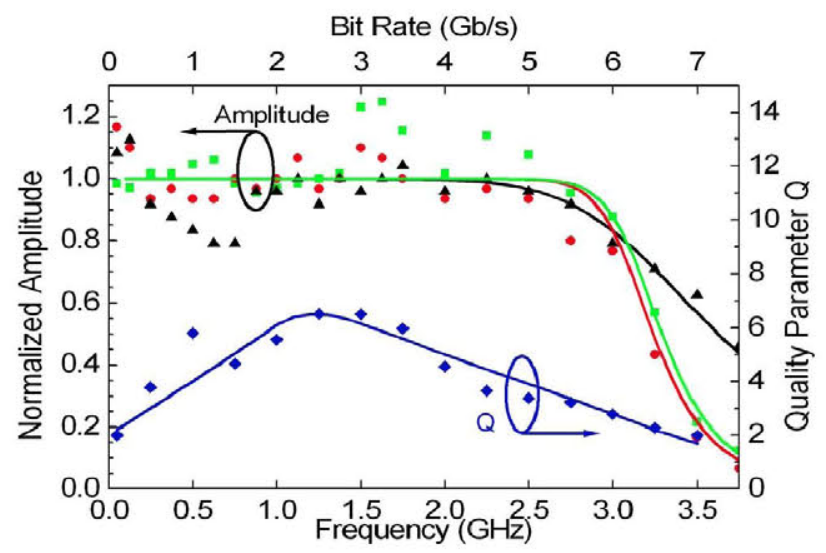

Fig. 4. Normalized large signal modulation response under square modulation for different modulation depths, corresponding to different peak-to-peak PPG output voltages: black triangles, $2.5 \mathrm{~V}$ (not amplified); red circles, amplified $(\sim \times 10) 0.25 \mathrm{~V}$; green squares, amplified $(\sim \times 10) 0.6 \mathrm{~V}$. The quality parameter $\mathrm{Q}$ corresponding to the amplified $0.6 \mathrm{~V}$ modulation depth is shown by the blue diamonds. The lines are drawn as a visual aid.
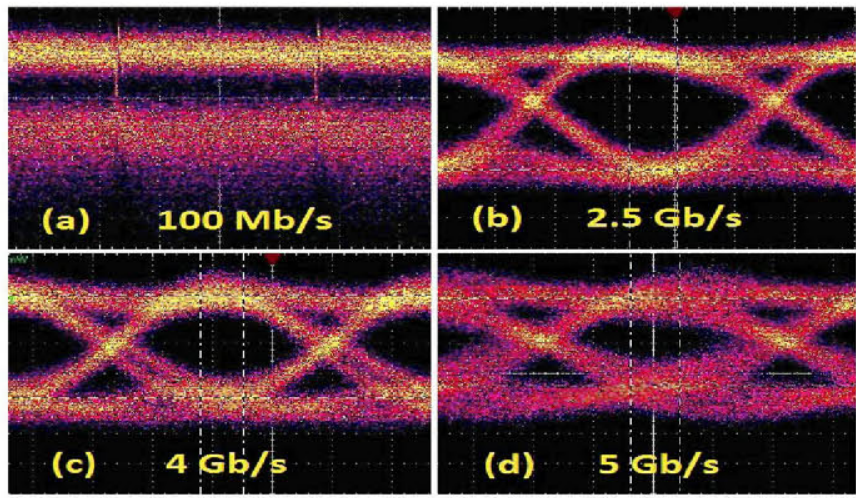

Fig. 5. Eye diagrams measured at tapered current of $4 \mathrm{~A}$, RW current of $120 \mathrm{~mA}$, modulation amplitude of about $110 \mathrm{~mA}: 100 \mathrm{Mb} / \mathrm{s}(\mathrm{a}), 2.5 \mathrm{~Gb} / \mathrm{s}$ (b), $4 \mathrm{~Gb} / \mathrm{s}(\mathrm{c})$, and $5 \mathrm{~Gb} / \mathrm{s}(\mathrm{d})$.

about $2.5 \mathrm{~V}$ generated by the PPG $(0.25 \mathrm{~V})$ and amplified $(\sim \times 10)$ by the RF amplifier. For high frequencies the OMA falls faster and the cut-off frequency is lower when using the RF amplifier. Actually, the amplitude response of the system with RF amplifier falls to $50 \%$ at around $3.3 \mathrm{GHz}$, while without RF amplifier it falls to the same level at around 3.7 GHz. This indicates that it is the RF amplifier rather than the laser itself what is actually limiting the frequency response of our system.

The good high frequency response $(>3.5 \mathrm{GHz}$ ) of this long cavity laser was not expected, specially taking into account that the contact layout was designed for $\mathrm{CW}$ operation. We attribute the good performance to the dynamics of multisection lasers, which can strongly enhance the modulation bandwidth [11 and references herein]. Further theoretical and experimental research is still necessary for a proper understanding of the dynamic properties of multi-section tapered lasers.

Fig. 4 also shows the dependence of the quality parameter $\mathrm{Q}$ on the bit rate when using amplified $0.6 \mathrm{~V}$ driving voltage. The estimated peak to peak current excursion was $110 \mathrm{~mA}$. The value of $Q$ improves with the data rate reaching a maximum of around 6.5 (corresponding to a BER $\leq 10^{-10}$ ) and degrades again at higher data rates. It is important to remark that the noise observed in the eye diagrams (see Fig. 5) is basically due to the laser dynamics itself and not to the detector noise. The low value of $Q$ at low data rates is a consequence of the low frequency cut-off of the electrical preamplifier $(10 \mathrm{MHz})$. This reduces the amplitude of the driving signal after long sequences of zeros or ones (up to 31 bits). Since the same sequences of zeros or ones are longer in time for lower $B_{R}$ the degradation effect on the eye diagrams is more relevant at low $B_{R}$, as it can be observed in Fig. 5 a at $B_{R}=100 \mathrm{Mb} / \mathrm{s}$. Fig. 5 b) shows a clearly open eye-diagram at $2.5 \mathrm{~Gb} / \mathrm{s}$ with a high OMA of $0.53 \mathrm{~W}$, a modulation efficiency of around $5 \mathrm{~W} / \mathrm{A}$, and an ER of around $3 \mathrm{~dB}$. At data rates higher than $3 \mathrm{~Gb} / \mathrm{s}$ the opening of the eye diagram is initially limited by patterning effects, as shown in Fig. 5c) at $4 \mathrm{~Gb} / \mathrm{s}$, where a double switch-off trace can be appreciated. At higher data rates the high frequency response of the amplifier and the associated time jitter degrades the eye-diagram (see Fig. $5 \mathrm{~d}$ at $5 \mathrm{~Gb} / \mathrm{s}$ ).

\section{CONCLUSION}

In summary, the direct modulation of a high power separate contact tapered laser emitting at $1060 \mathrm{~nm}$ at data rates up to $2.5 \mathrm{~Gb} / \mathrm{s}$ with a large modulation amplitude of $0.53 \mathrm{~W}$ has been demonstrated. The results indicate that the maximum measured bit rate is limited by the driving circuit rather than by the intrinsic laser dynamics and that higher data rates could be achieved in an improved set-up. These achievements pave the way to the application of directly modulated two section tapered lasers in free-space optical communications.

\section{REFERENCES}

[1] H. Henniger and O. Wilfret, "An introduction to free-space optical communications," Radioengineering, vol. 19, no. 2, pp. 203-212, Jun. 2010.

[2] G. Blume, D. Feise, C. Kaspari, A. Sahm, and K. Paschke, "High luminance tapered diode lasers for flying-spot display applications," Proc. SPIE, vol. 8280, pp. 82800 E-1-82800E-10, Jan. 2012.

[3] X. Ai, R. Nock, J. G. Rarity, and N. Dahnoun, "High-resolution randommodulation CW lidar," Appl. Opt., vol. 50, no. 22, pp. 4478-4488, Aug. 2011.

[4] K. Paschke, et al., "Nearly diffraction limited 980-nm tapered diode lasers with an output power of 7.7 W," IEEE J. Sel. Topics Quantum Electron., vol. 11, no. 5, pp. 1223-1227, Sep./Oct. 2005.

[5] N. Michel, et al., "High modulation efficiency and high power $1060 \mathrm{~nm}$ tapered lasers with separate contacts," Electron. Lett., vol. 45, no. 2, pp. 103-104, Jan. 2009.

[6] C. H. Kwok, R. V. Penty, I. H. White, K.-H. Hasler, B. Sumpf, and G. Erbert, "High modulation efficiency Gigabit/s modulation of twincontact high-brightness tapered DBR laser," IEEE Photon. Technol. Lett., vol. 21, no. 5, pp. 301-303, Mar. 1, 2009.

[7] K.-H. Hasler, et al., "Improving the modulation efficiency of high-power distributed Bragg reflector tapered diode lasers," Appl. Phys. B, vol. 102, no. 1, pp. 43-47, 2011.

[8] A. Klehr, B. Sumpf, K.-H. Hasler, J. Fricke, A. Liero, and G. Erbert, "High-power pulse generation in GHz range with 1064-nm DBR tapered laser," IEEE Photon. Technol. Lett., vol. 15, no. 11, pp. 832-834, Jun. 1, 2010.

[9] B. Sumpf, et al., "1060 nm DBR tapered lasers with $12 \mathrm{~W}$ output power and a nearly diffraction limited beam quality," Proc. SPIE, vol. 7230, pp. 72301E-1-72301E-8, Feb. 2009.

[10] C. Fiebig, V. Z. Tronciu, M. Lichtner, K. Paschke, and H. Wenzel, "Experimental and numerical study of distributed-bragg-reflector tapered lasers," Appl. Phys. B, vol. 99, nos. 1-2, pp. 209-214, Apr. 2010.

[11] N. G. Usechak, M. Grupen, N. Naderi, Y. Li, L. F. Lester, and V. Kovanis, "Modulation effects in multi-section semiconductor lasers," Proc. SPIE, vol. 7933, pp. 79331I-1-79331I-14, Jan. 2011. 